\title{
Estratégia para Melhoria de Processos em Conformidade com o CMMI e o MR mps Br no BNDES
}

\author{
Sérgio Marques de Viveiros ${ }^{1}$, Cássio Adriano Nunes Teixeira ${ }^{1,2}$, Jorge Luiz Dias \\ Ramos ${ }^{1}$, Ariana Ribeiro Gomes de Assumpção ${ }^{1}$, Ana Regina Rocha ${ }^{2}$ \\ ${ }^{1}$ BNDES - Banco Nacional de Desenvolvimento Econômico e Social \\ smv@bndes.gov.br, cassio@bndes.gov.br,argda@bndes.gov.br, jlram@bndes.gov.br \\ ${ }^{2}$ COPPE/UFRJ - Programa de Engenharia de Sistemas e Computação \\ cant@cos.ufrj.br, darocha@cos.ufrj.br
}

\begin{abstract}
In this paper we present a software process improvement project being conducted in BNDES, the Brazilian Development Bank, along with its objectives, guidelines and strategies. We also describe the scenario that made the project necessary and present some results already achieved.
\end{abstract}

Resumo. Este artigo apresenta um projeto de melhoria de processo de software que vem sendo conduzido no Banco Nacional de Desenvolvimento Econômico e Social - BNDES, seus objetivos, diretrizes e estratégias. Descrevemos também o cenário que motivou sua existência e os resultados obtidos até o momento.

\section{Introdução}

Embora seja uma área de conhecimento recente se comparada à Administração ou ao Direito, a Tecnologia da Informação (TI) já é atualmente um fator estratégico na maior parte das empresas públicas e privadas. A qualidade da informação e a agilidade para responder às demandas do mercado são hoje fatores críticos de sucesso para qualquer empresa. Embora exerça um papel fundamental para permitir o alcance destes objetivos, a TI muitas vezes se encontra em um baixo estágio de maturidade nas organizações. É comum encontrarmos nas empresas equipes de desenvolvimento que não seguem processos definidos e conduzem seus projetos de modo informal.

A situação atual da TI no BNDES não é muito diferente da maioria das empresas brasileiras - não é seguido um processo padronizado de desenvolvimento de sistemas. Cada gerente conta apenas com sua própria experiência para conduzir os projetos sob sua responsabilidade e o sucesso depende diretamente das individualidades envolvidas. Não há documentação mínima exigida, nem lista de produtos obrigatórios para que os sistemas sejam homologados. O relacionamento com os clientes não é registrado nem formalizado devidamente. Essa situação causa uma série de problemas: inexistência de métricas, previsões irreais e compromissos não mantidos, dificuldade na gerência dos projetos, perda de produtividade, aumento dos esforços de manutenção e retrabalho, dificuldade de utilização e compartilhamento do conhecimento gerado por TI, conflitos com os clientes e entrega de produtos inadequados às demandas da instituição.

Na tentativa de reverter esta situação, em março de 2003 foi criada a Gerência de Processo de Engenharia de Software (DEINP/GPES), cuja tarefa primordial é implantar um processo de software para o Departamento de Desenvolvimento e Manutenção de Sistemas: o DESIS. 
Ao longo de 2003 a equipe DEINP/GPES se capacitou, efetuou um diagnóstico dos principais problemas do desenvolvimento de sistemas no BNDES e realizou o planejamento do Projeto de Implantação de Processo de Software do BNDES (IPSBNDES) Com base no diagnóstico realizado, decidiu-se adotar o CMMI - Capability Maturity Model Integration - (CMMI, 2002) como guia para o projeto. Paralelamente, foram realizados contatos com diversas instituições visando à contratação de uma consultoria especializada, o que em agosto de 2004 culminou com a contratação da COPPE/UFRJ através da Fundação COPPETEC, por sua reconhecida experiência nessa área. Ainda em 2004, com a proposta do MR mps Br (Modelo de Referência para melhoria do processo de software Brasileiro - veja detalhes em: WEBER, et al. (2004)) a aderência a este modelo foi incorporada aos objetivos do Projeto IPS-BNDES.

Este artigo descreve o projeto IPS-BNDES e seus objetivos, detalhando a estratégia definida para a definição e implantação do processo. Descreve também o estágio atual do projeto e suas perspectivas futuras.

\section{Objetivos do Projeto IPS-BNDES}

O Projeto IPS-BNDES é fruto de uma forte demanda do DESIS em trabalhar com mais qualidade, de forma padronizada, porém sem tirar a criatividade de seus profissionais. $\mathrm{O}$ compromisso do projeto é implantar com sucesso um processo de software adequado à realidade do BNDES, aprimorando a qualidade dos produtos, formalizando $\mathrm{e}$ melhorando a relação com seus clientes. O projeto tem os seguintes objetivos:

- Melhorar a qualidade dos produtos de software desenvolvidos e mantidos pelo BNDES;

- Definir e implantar no DESIS processos padrão relacionados às áreas de processo (PAs) do CMMI Níveis 2 e 3, compatíveis com o MR mps Br;

- Consolidar a DEINP/GPES como gestora do processo de software no BNDES;

- Disponibilizar para o DESIS ferramentas de apoio à execução dos processos;

- Disseminar os processos no DESIS, treinando e preparando todos os envolvidos;

- Disponibilizar os processos, artefatos e informações para acompanhamento dos projetos do DESIS em um portal na intranet;

- Possibilitar que o conhecimento de TI esteja registrado de forma organizada (documentado em artefatos) e que possa ser reutilizado;

- Diminuir a dependência do conhecimento individual dos analistas sobre os sistemas, fazendo com que o conhecimento adquirido com o desenvolvimento e a manutenção dos produtos possa ser registrado e compartilhado;

- Permitir a gradativa redução do esforço de manutenção de sistemas legados no mainframe.

Para atingir estes objetivos o projeto IPS segue uma estratégia que prioriza as características do BNDES, especialmente o fato deste ser uma grande empresa pública e estar prestes a adotar um modelo de prestação de serviços de desenvolvimento através de fábricas de software.

\section{O cenário}

O DESIS é um departamento subordinado à Área de Controle do BNDES e possui 11 gerências, que respondem pelos projetos e sistemas. As gerências fazem também o papel de interlocução com as 14 áreas do BNDES, fazendo com que cada gerência 
atenda a pelo menos uma área. Com uma equipe de cerca de 60 profissionais e igual (por vezes superior) número de terceirizados, o DESIS tem como principais atribuições:

- manter interlocução com as Unidades Fundamentais para atendimento de suas necessidades relativas à tecnologia da informação;

- projetar, desenvolver, implantar e manter os sistemas aplicativos do Sistema BNDES, e elaborar e manter atualizada a documentação desses sistemas;

- dimensionar, avaliar e prover soluções (inclusive avaliando soluções de mercado) de aplicativos adequadas às necessidades do Sistema BNDES;

- elaborar as especificações de projeto dos sistemas aplicativos com o objetivo de garantir a sua segurança;

- administrar os serviços contratados de terceiros sob sua responsabilidade.

\section{Algumas dificuldades e as soluções vislumbradas}

Qualquer projeto de melhoria de qualidade de software, se quiser lograr êxito, deve levar em conta recomendações sobre a importância de fatores "não técnicos":

Gerenciamento efetivo trata da gerência das pessoas na organização. Gerentes de projetos têm que resolver problemas técnicos e não técnicos através das pessoas alocadas em suas equipes da maneira mais efetiva possível (SOMMERVILLE, 2004, p.592, grifo nosso).

Dentre outras, é comum encontrar as seguintes necessidades ressaltadas: robusto patrocínio para o projeto; comunicação efetiva; programas de incentivo atrelado ao uso do processo; considerações sobre o clima organizacional; envolvimento daqueles cuja relação com o trabalho será alterada pelo processo (McFEELEY, 1996). São comuns informações sobre reveses em projetos de melhoria de qualidade de software em decorrência de questões culturais, políticas e organizacionais. Dificilmente um projeto desses malogra em função de questões técnicas. Por exemplo, Fuggeta (2000), se referindo ao CMM - Capability Maturity Model, nos alerta para os riscos de ignorarmos questões que podem ser críticas para o projeto porque a maioria das indicações sugeridas por este modelo foca apenas aspectos de engenharia.

Sensíveis a esses alertas, deparávamo-nos com os seguintes problemas: o Projeto IPS-BNDES não dispunha de patrocínio adequado - existiam apenas afirmações não contundentes de que processo de software "era importante"; não dispúnhamos do apoio de um "campeão" - não existia nenhum líder natural no grupo, que a DEINP/GPES pudesse tomar como exemplo na definição / uso do processo; o tempo de vida médio da gerência sênior era muito reduzido. Não bastasse, na realidade de uma empresa pública quase ninguém se sentiria ameaçado pelo processo vindouro - por perverso que pareça, forçoso é reconhecer que a possibilidade de demissão existente na iniciativa privada traz grande estímulo a que os desenvolvedores utilizem o processo definido.

Não existe dúvida sobre algumas pessoas sentirem-se ameaçadas pelas mudanças e preocupadas em perderem seus empregos ou não se adequar às novas formas de trabalho. É necessário envolver o time ao longo de todo o processo de mudança, entendendo suas dúvidas e envolvendo-os no planejamento do novo processo [de software]. Tornando-os stakeholders no processo de mudança, é muito mais provável que eles desejarão realizar o trabalho (SOMMERVILLE, 2004, p.680, grifo nosso).

Estes vários fatores muito cedo fizeram surgir a premissa mais forte do Projeto IPS: envolver os desenvolvedores induzindo-os, eles próprios, a estabelecerem o processo de software. Mesmo porque: 


\begin{abstract}
Melhoria de processo [de software] não significa simplesmente adotar métodos e ferramentas particulares ou usar algum modelo de processo utilizado em outro lugar. Embora organizações que desenvolvem o mesmo tipo de software claramente têm muito em comum, existem sempre fatores, procedimentos e padrões locais que influenciam o processo. Raramente será bem sucedido na introdução de melhorias no processo aquele que simplesmente tentar mudar o processo [local] para algum outro utilizado em outro lugar. Sempre deve-se olhar para melhoria de processo como algo específico para a organização (SOMMERVILLE, 2004, p.666).
\end{abstract}

Buscando envolver e aliar os desenvolvedores, criamos um grupo representativo, composto pelo chefe e alguns executivos do DESIS, chamado de Grupo Homologador (GH). Trata-se de um management steering group (McFEELEY, 1996, 216) que vincula o Projeto IPS às necessidades e visão do DESIS. Esse grupo é o interlocutor formal do DESIS para o projeto - toda demanda ou alteração deve ser autorizada por ele. Adicionalmente, o GH é responsável pela aceitação dos produtos do projeto IPSBNDES e pela definição das atividades de treinamento.

O Projeto IPS-BNDES é de longo prazo, mais de quatro anos. Para reduzir o risco de ser abortado sem gerar benefício algum, foi planejado para produzir resultados intermediários a cada quatro meses, requerendo, portanto, uma criteriosa priorização das áreas de processo (PAs) do CMMI que seriam abordadas.

A partir desse cenário, configura-se o tripé do Projeto IPS: o processo deveria ser definido participativamente; a implantação do processo deveria ser gradual; e o processo seria baseado no CMMI. O CMMI-SE/SW foi definido como o pano de fundo do projeto e guia para a definição do processo de software do BNDES, porque propõe caminho gradual de melhoria a ser definido pela própria empresa (CHRISSIS, 2003). O Projeto IPS, entretanto, não busca neste momento a avaliação oficial CMMI, por não ser o BNDES uma empresa de desenvolvimento de software. Todavia, está prevista a realização de avaliações MR mps BR, pelos seguintes motivos: investimento mais acessível em relação ao CMMI; visibilidade de resultados mais rápida em comparação ao CMMI, por conta da existência de mais níveis de maturidade no MR mps BR; e a possibilidade de servir de exemplo de esforço de qualidade no mercado de software brasileiro, incentivando a adoção do MR mps BR em outras empresas brasileiras.

\title{
5. Estratégia para Definição e Implantação dos Processos
}

Após a contratação da COPPE/UFRJ foi detalhado o planejamento do projeto IPS, que envolveu o refinamento da estratégia elaborada para definição e implantação dos processos, considerando os objetivos da empresa.

O CMMI-SE/SW se estrutura em áreas de processo (PAs). Fazem parte do escopo do projeto IPS 18 PAs, que foram divididas em quatro blocos:

- Bloco 1 - contém as PAs: Planejamento de Projeto, Monitoração e Controle de Projeto, Gerência de Requisitos e Gerência de Acordos com Fornecedores. As duas PAs relacionadas à gerência de projetos (Planejamento de Projetos e Monitoração e Controle de Projetos) são ponto de partida bastante adequado em projetos deste tipo, pois abordam práticas que devem ser usadas em qualquer projeto de TI (mesmo que não seja de desenvolvimento). De fato, o BNDES já vem investindo em treinamento em gerência de projetos para os seus executivos, reforçando ainda mais a necessidade de colocar em prática os conhecimentos adquiridos. A PA Gerência de Requisitos também é uma opção natural, pois aborda o clássico problema de garantir um compromisso entre TI e clientes 
sobre os requisitos que serão atendidos e a difícil gerência das mudanças nos requisitos ao longo do projeto. O CMMI sugere que essas três PAs sejam as primeiras a serem tratadas, e elas compõem o primeiro nível de maturidade do MR mps Br - Nível G - Parcialmente Gerenciado. A inclusão da PA Gerência de Acordos com Fornecedores neste bloco se dá por causa da perspectiva do DESIS vir trabalhar com fábricas de software num futuro próximo.

- Bloco 2 - contém as PAs: Gerência de Configuração, Medição e Análise, Gerência da Qualidade do Processo e do Produto, Gerência de Riscos, Foco no Processo Organizacional e Definição do Processo Organizacional. Este bloco encerra o Nível 2 do CMMI-SE/SW em estágios (e também o Nível F do MR mps BR) e avança por algumas PAs do Nível 3, fazendo com que os projetos do DESIS utilizem um processo padronizado. A PA Gerência de Riscos (presente no Nível $\mathrm{C}$ do MR mps BR) foi priorizada por sua proximidade com as PAs gerenciais, e as PAs Foco no Processo Organizacional e Definição do Processo Organizacional (presentes no Nível D do MR mps BR) já estarão sendo atacadas parcialmente ao longo do projeto, uma vez que toda homologação de PA estabelece um pedaço do processo de software padrão do BNDES, e a manutenção desse processo é responsabilidade da própria equipe do projeto IPS.

- Bloco 3 - contém as PAs: Desenvolvimento de Requisitos, Solução Técnica, Verificação, Validação e Integração do Produto. Este bloco aborda as PAs de Engenharia e coincide com o Nível E do MR mps BR. Acreditamos que a experiência acumulada nos primeiros blocos permitirá criar um ambiente propício à adoção de técnicas, etapas e artefatos de engenharia padronizados no DESIS, fato que não ocorreria no início do projeto.

- Bloco 4 contém as PAs: Análise de Decisão e Resolução, Gerência de Projetos Integrada e Treinamento Organizacional. Este bloco alcança o Nível 3 do CMMI-SE/SW e os Níveis D e C do MR mps BR, completando o escopo do Projeto IPS, que não prevê a definição e implantação de processos relacionados aos níveis 4 e 5 do CMMI ou aos níveis A e $\mathrm{B}$ do MR mps Br.

O fator Treinamento, considerado crítico para o sucesso do projeto, se iniciou com 11 palestras ministradas pela COPPE/UFRJ abordando os seguintes temas: Engenharia de Software, Processos de Software, Qualidade de Produtos, Engenharia de Requisitos, Pontos de Função, Medição e Análise, Gerência de Configuração, Inspeção de Software, Testes de Software, Gerência de Acordos com Fornecedores e Gerência do Conhecimento. Estas palestras marcaram o início da execução do projeto e tiveram horários alternativos para facilitar a participação de toda a equipe de TI do BNDES.

Estabelecidos os quatro blocos e as áreas de processo que seriam tratadas em cada bloco, passou-se à definição dos passos a serem seguidos para sua implementação. Buscou-se, então, definir uma estratégia que envolvesse o máximo possível todos os profissionais do DESIS, fazendo uso de seu conhecimento e experiência para garantir a qualidade e adequação dos processos definidos, e que, ao mesmo tempo, garantisse a produtividade na definição e implantação dos processos na empresa. Desta forma foi estabelecido o seguinte procedimento para definição e implantação do processo:

1. Treinamento prévio da equipe DEINP/GPES e do Grupo Homologador (GH) sobre a PA - Realizada pela consultoria da COPPE, esta atividade consiste 
de uma reunião de 2 horas para discutir a PA em questão com o $\mathrm{GH}$ e a DEINP/GPES. A discussão exige a leitura prévia do texto oficial do CMMI.

2. Definição inicial de um processo para a PA - Realizada pela consultoria da COPPE, a atividade consiste na definição inicial e simplificada de um conjunto de atividades que é entregue à DEINP/GPES e serve de ponto de partida para o trabalho de definição de processo para atender à PA.

3. Treinamento conceitual na PA para toda equipe do DESIS - Realizada pela consultoria da COPPE, a atividade consiste de uma palestra de 2 horas que apresenta a PA em questão a toda a equipe do DESIS. Realizada em dois horários alternativos, a palestra visa a fornecer um embasamento mínimo aos desenvolvedores para que possam opinar sobre o processo que será proposto.

4. Definição do processo para a PA pelo BNDES - Esta atividade é realizada pela DEINP/GPES que, a partir da definição inicial e usando seus conhecimentos da instituição e da PA em questão, define o processo do BNDES. O processo definido contém políticas, procedimentos, etapas, atividades, artefatos, em suma, todo o detalhamento para sua execução no BNDES. Ao término desta atividade, o GH avalia o processo proposto e delibera se já está adequado para submissão às críticas do DESIS.

5. Análise do processo pela equipe do DESIS - A participação do corpo funcional é fundamental para o sucesso do Projeto IPS. Em função disso, os produtos propostos passam obrigatoriamente por uma etapa de discussão com todos os desenvolvedores. Isso se dará sempre a partir de propostas da DEINP/GPES, através de reuniões e lista eletrônica de discussão, com prazo definido de encerramento. As contribuições obtidas da lista de discussão são processadas pela DEINP/GPES e discutidas com o GH.

6. Reunião de consenso com a consultoria - Após a discussão com o DESIS, e feitas as devidas alterações pela DEINP/GPES, o material é enviado à consultoria da COPPE, que se reúne com a DEINP/GPES para discutir o resultado e garantir a aderência do processo ao CMMI e ao MR mps BR.

7. Homologação da PA - Após a execução das etapas anteriores, a PA é homologada através de um instrumento oficial interno ao BNDES.

8. Realização de um projeto piloto - Após a homologação de um processo para uma PA, um projeto piloto será executado para avaliar o processo proposto. Os pilotos terão necessariamente uma fase de encerramento, na qual serão registradas as sugestões de mudanças para o processo e seus artefatos.

9. Aceite Final da PA - Após avaliação das sugestões de mudanças derivadas do piloto, a DEINP/GPES e o GH deliberam sobre o que será incorporado ao processo da PA e implementam as mudanças.

10. Capacitação do DESIS no processo relativo à $\mathrm{PA}$ - O processo da $\mathrm{PA}$ já estava homologado e, portanto, já devia estar sendo seguido. A institucionalização efetiva ocorre após o piloto, com o treinamento de todos os desenvolvedores envolvidos com o processo relativo à PA.

Chamamos a atenção para a ocorrência da homologação antes da execução do piloto. A escolha dos pilotos não está sob domínio completo da DEINP/GPES, existindo 
grande incerteza sobre seu planejamento. Para não impactar o IPS, optou-se por uma estratégia em que os pilotos seguem paralelamente à execução de outras atividades no projeto. Assim, imediatamente após uma homologação, existindo ou não possibilidade de iniciar um piloto, a DEINP/GPES inicia o ciclo de uma nova PA. O risco do processo de uma PA se mostrar inadequado à realidade do BNDES fica mitigado pela participação do grupo de desenvolvedores na definição do processo.

Desde seu planejamento, o projeto IPS-BNDES definiu com seu cliente - o DESIS - os critérios para avaliação de sua conclusão:

1. Encerramento de pelo menos um piloto para cada PA.

2. Disponibilização de um site com os processos de todas as PAs.

3. Encerramento de todos os treinamentos relacionados à PAs.

4. Institucionalização (transformação em regras) de todas as PAs previstas.

5. Definição de responsáveis para os processos relativos a todas PAs.

\section{Estágio Atual e Perspectivas Futuras}

O Projeto IPS-BNDES se encontra em andamento com um conjunto de atividades já realizadas e resultados obtidos que comentamos a seguir.

Como previsto, o projeto teve início com a realização das seguintes atividades: (i) formação do $\mathrm{GH}$, (ii) realização das atividades iniciais de treinamento e (iii) definição dos processos Planejamento de Projetos e Monitoração e Controle de Projetos.

A formação e o envolvimento do $\mathrm{GH}$ vem sendo um fator crítico de sucesso. A partir de um treinamento específico para o grupo em cada área de processo, visando um conhecimento aprofundado, o grupo tem participado da definição e validação dos processos até a sua homologação. A estratégia do projeto, neste aspecto, tem se mostrado muito adequada e com excelentes resultados.

As atividades iniciais de treinamento foram realizadas através de 11 palestras com duração de 2 a 4 horas, oferecidas em horários alternativos, permitindo a participação de toda a equipe. As palestras foram, em geral, avaliadas positivamente pelos participantes quanto ao seu conteúdo e importância para o projeto IPS-BNDES. Também já foram realizadas palestras sobre as duas PAs iniciais do projeto.

Finalmente, foram iniciadas as atividades que envolvem a definição e a homologação dos processos com os processos Planejamento de Projetos e Monitoração e Controle de Projetos. Foram realizadas as sete primeiras atividades e neste momento está sendo escolhido um projeto piloto. Os passos anteriores foram realizados de forma totalmente satisfatória envolvendo toda a equipe do DESIS, além do GH.

As perspectivas para o futuro imediato são realizar as atividades para a PA Gerência de Requisitos e em seguida realizar a avaliação do mps Br Nível G.

\section{Conclusão}

Neste trabalho, relatamos a experiência de melhoria de processo de software no BNDES, o Projeto IPS-BNDES. Através deste projeto a instituição passará a contar com um processo padronizado de software, e cada novo projeto de desenvolvimento o terá como base para seu planejamento e realização. Por definição, todo projeto tem sempre suas diferenças e particularidades, e os gerentes poderão fazer as adaptações necessárias 
ao seu problema específico. Assim, o processo padronizado não se transforma numa "camisa-de-força", mas num guia prático seguido por todos e adaptado a cada projeto.

Com processos padronizados, será possível medir e comparar os projetos entre si. Assim que essas medições comecem a gerar um histórico dos projetos de TI na empresa, pode-se passar a prever os parâmetros dos projetos com melhor precisão. Com erros menores de prazo e custo, melhora-se o relacionamento com os clientes e aumenta-se a credibilidade da TI na instituição. Padronizar os processos também é muito útil para a TI: fica mais simples gerenciar os projetos e alocar os recursos, já que os projetos se tornam mais previsíveis. De forma similar, processos padronizados deixam os projetos mais transparentes - os clientes têm melhores condições de entender o que acontece no projeto, acompanhar suas etapas e programar seu envolvimento. $\mathrm{O}$ resultado é a participação mais efetiva dos clientes, a redução de conflitos e a identificação precoce de problemas, reduzindo custos nos projetos. Com menos problemas no projeto e menos erros nos sistemas entregues, a TI atinge melhores índices de produtividade e consegue resolver as demandas mais rapidamente, criando um círculo virtuoso na empresa.

Os passos já realizados mostraram a viabilidade e a adequação da estratégia incremental e participativa para o BNDES. Acreditamos que ela possa ser facilmente adaptada às necessidades de outras empresas públicas de grande porte.

\section{Referências}

CHRISSIS, M., KONRAD, M., SHRUM, S., 2003, CMMI: Guidelines for Process Integration and Product Improvement. Addison-Wesley.

CMMI, 2002, Capability Maturity Model Integration, Version 1.1: Staged representation. Technical Report, CMU/SEI-2002-TR-012, Software Engineering Institute, Pittsburgh, Pennsylvania, EUA.

FUGGETTA, A., 2000, "Software Process: A Roadmap". In: FINKELSTEIN, A. (ed.), The Future of Software Engineering.

McFEELEY, B., 1996, IDEAL: A User's Guide for Software Process Improvement. Handbook, CMU/SEI/96-HB-001, feb.

SOMMERVILLE, I., 2004, Software engineering. $7^{\text {th }}$ ed., Addson-Wesley.

WEBER, K.C., 2004, "Modelo de Referência para Melhoria de Processo de Software: uma abordagem brasileira." In: Conferência Latinoamericana de Informática (CLEI2004), 30, 2004, Arequipa Peru. (Disponível em: http://www.softex.br/ media/artigoCLEI_versao_final.pdf. Acesso em 28 fev. 2005) 